\title{
Infinite-range Ising ferromagnet in a time-dependent transverse magnetic field: quench and ac dynamics near the quantum critical point
}

\author{
Arnab Das ${ }^{(1)}$, K. Sengupta(1), Diptiman Sen ${ }^{(2)}$ and Bikas K. Chakrabarti(1) \\ (1) Theoretical Condensed Matter Physics Division and Center for Applied Mathematics and Computational Science, \\ Saha Institute of Nuclear Physics, 1/AF Bidhannagar, Kolkata 700064, India \\ ${ }^{(2)}$ Center for High Energy Physics, Indian Institute of Science, Bangalore 560012, India
}

(Dated: October 2, 2018)

\begin{abstract}
We study an infinite range ferromagnetic Ising model in the presence of a transverse magnetic field which exhibits a quantum paramagnetic-ferromagnetic phase transition at a critical value of the transverse field. In the thermodynamic limit, the low-temperature properties of this model are dominated by the behavior of a single large classical spin governed by an anisotropic Hamiltonian. Using this property, we study the quench and AC dynamics of the model both numerically and analytically, and develop a correspondence between the classical phase space dynamics of a single spin and the quantum dynamics of the infinite-range ferromagnetic Ising model. In particular, we compare the behavior of the equal-time order parameter correlation function both near to and away from the quantum critical point in the presence of a quench or AC transverse field. We explicitly demonstrate that a clear signature of the quantum critical point can be obtained by studying the $\mathrm{AC}$ dynamics of the system even in the classical limit. We discuss possible realizations of our model in experimental systems.
\end{abstract}

PACS numbers: $73.43 . \mathrm{Nq}, 05.70 . \mathrm{Jk}$

\section{INTRODUCTION}

Quantum phase transitions have been studied extensively for several systems ${ }^{1}$. More often than not, simple prototype systems such as the Ising model in a transverse field or rotor models bring out many important characteristics of such transitions 1.2 . In most of these studies so far, only the equilibrium situation, where the system is taken adiabatically across the quantum critical point (QCP), has been addressed ${ }^{1.2}$. It is well known that in such cases, the thermodynamic properties of the system can be charted by determining the critical exponents and hence the universality class of the transition ${ }^{1.2 .3}$. However, no such general criteria exists for non-equilibrium dynamics of quantum critical systems.

In recent years, there has been both theoretical and experimental progress in understanding the behavior of non-equilibrium dynamics near QCPs. On the experimental side, there have been several experiments on ultra-cold atoms in optical lattices which realize such non-equilibrium situations ${ }^{4}$. On the theoretical side, there have been studies of one-dimensional Ising models in a transverse field and their variants $\stackrel{5}{\underline{5}}$, driven quantum spin chains $\boldsymbol{6}^{\underline{6}}$, and correlation functions for quench dynamics close to a quantum critical point ${ }^{7}$. The main additional difficulty over the equilibrium case that one encounters in these theoretical studies arises from the fact that the description of non-equilibrium dynamics near a QCP necessitates the knowledge of all or at least the first few excited states in the Hilbert space of the manybody Hamiltonian. This precludes a detailed study of most systems which can be realized experimentally. The notable exceptions are situations where the systems can be described by either integrable models 5.6 , or can be analyzed numerically by exact diagonalization methods applicable to finite-size systems ${ }^{5}$.

In this work, we study both the quench and the AC dynamics of an infinite-range ferromagnetic Ising model in the presence of a transverse field near its QCP. Such a model, as we show in this work, can be represented by a single large spin. This particular feature of the model allows us to analyze its non-equilibrium dynamics near the QCP. Further, the value of this single spin increases linearly with the system size so that the large spin and thermodynamic limits coincide for the model. This enables us to describe the system in the thermodynamic limit accurately using classical equation of motions for a single spin. In particular, we can develop a correspondence between the classical phase space dynamics of a single spin and the quantum dynamics of the infinite-range ferromagnetic Ising model in the presence of a transverse field. In view of the long range dipoledipole interactions involved in many systems with orderdisorder transitions driven by tunneling fields, the study of quantum critical behavior of this model is not just a matter of theoretical curiosity, but can have application in ferroelectrics ${ }^{2}$ like $\mathrm{KH}_{2} \mathrm{PO}_{4}$ and ferromagnets like Dy $\left(\mathrm{C}_{2} \mathrm{H}_{5} \mathrm{SO}_{4}\right)_{3} 9 \mathrm{H}_{2} \mathrm{O}$. Further this model can also can be realized in two-component Bose-Einstein condensates (BEC) of ultracold atoms ${ }^{8}$.

The main results reported in this work are the following. First, we obtain the equilibrium phase diagram for the infinite range model and also obtain its collective excitations using a Holstein-Primakoff approach for both the ferromagnetic and the paramagnetic phase. Second, we study the quench dynamics of the equal-time order parameter correlation function (EOC) and obtain analytical expressions for its long-time behaviors. Finally, we study the dynamics of the EOC in the presence of an weak external AC transverse field; we show that the response of 
the system in the presence of the AC field near the QCP involves multiple frequencies and hence appears noisy in the time domain. This behavior, which persists even in the classical large $S$ limit of the model, is to be contrasted to that in both the paramagnetic and the ferromagnetic phases far from the critical point where the response of the system involves only a few frequencies and does not exhibit such noisy behavior. There are some studies on classical Ising models in the presence of oscillating longitudinal fields ${ }^{9}$ and some mean field and Monte Carlo studies $\frac{10.11}{1}$ in the presence of an oscillating transverse field. However, these studies are for completely different models and none of them investigates the true quantum dynamics.

The organization of the paper is as follows. In Sec. III we introduce the model, chart out its phase diagram and study its equilibrium properties. In Sec. III where we discuss the quench dynamics of the system across the QCP both classically and quantum mechanically. In Sec. IV we study the AC dynamics of the model. This is followed in Sec. $\nabla$ by a discussion of possible realizations of our model in experimental systems and conclusions.

\section{EQUILIBRIUM PROPERTIES}

We consider a system of $N$ spin- $1 / 2$ objects governed by the Hamiltonian

$$
H=-\frac{J}{N} \sum_{i<j} S_{i}^{z} S_{j}^{z}-\Gamma \sum_{i} S_{i}^{x},
$$

where $S_{i}^{a}=\hbar \sigma_{i}^{a} / 2, a=x, y, z$ are respectively the $x, y$ and $z$ components of the spin- $1 / 2$ operator represented by the standard Pauli spin matrices $\sigma^{a}$. Here we assume that $J \geq 0$ (ferromagnetic Ising interaction). This Hamiltonian is invariant under the $Z_{2}$ symmetry $S_{i}^{x} \rightarrow S_{i}^{x}$, $S_{i}^{y} \rightarrow-S_{i}^{y}$, and $S_{i}^{z} \rightarrow-S_{i}^{z}$. (The $Z_{2}$ symmetry would not be present if there was a longitudinal magnetic field coupling to $\sum_{i} S_{i}^{z}$ ). Note that the model in Eq. (11) differs from the one studied in Ref. 12, where the spins were taken to be living on two sub-lattices, with Ising interactions only between spins on different sub-lattices. We consider here the ferromagnetic case, for which the static quantum critical behavior is simple and easily derivable, while some significant features of its dynamic critical behaviors are also analytically tractable. We take $\Gamma \geq 0$ without loss of generality since we can always resort to the unitary transformation $S_{i}^{x} \rightarrow-S_{i}^{x}, S_{i}^{y} \rightarrow-S_{i}^{y}$ and $S_{i}^{z} \rightarrow S_{i}^{z}$, which flips the sign of $\Gamma$ but leaves $J$ unchanged. Eq. (11) can be written as

$$
\begin{aligned}
H & =-\frac{J}{2 N}\left(S_{t o t}^{z}\right)^{2}-\Gamma S_{t o t}^{x}, \\
S_{t o t}^{z} & =\sum_{i} S_{i}^{z}, \quad S_{t o t}^{x}=\sum_{i} S_{i}^{x},
\end{aligned}
$$

and we have dropped a constant $(J / 2 N) \sum_{i}\left(S_{i}^{z}\right)^{2}=J / 8$ from the Hamiltonian in Eq. (2). In the rest of this work, we shall use units $\hbar=1$.

\section{A. Mean Field Theory}

We begin with a mean field analysis of the thermodynamics of the model described by Eq. (11). Denoting the mean field value $m=\sum_{i}\left\langle S_{i}^{z}\right\rangle / N$, the Hamiltonian governing any one of the spins is given by

$$
h=-J m S_{t o t}^{z}-\Gamma S_{t o t}^{x} .
$$

This is a two-state problem whose partition function can be found at any temperature $T$. If $\beta=1 / k_{B} T$, we find that $m$ must satisfy the self-consistent equation

$$
m=\frac{J m}{2 \sqrt{\Gamma^{2}+J^{2} m^{2}}} \tanh \left(\frac{\beta \sqrt{\Gamma^{2}+J^{2} m^{2}}}{2}\right) .
$$

This always has the trivial solution $m=0$. In the limit of zero temperature, there is a non-trivial solution if $\Gamma<J / 2$, with $|m|=(1 / 2) \sqrt{1-4 \Gamma^{2} / J^{2}}$; the energy gap in that case is given by $J / 2$. If $\Gamma>J / 2$, we have $m=0$ and the gap is given by $\Gamma-J / 2$. Hence there is a zero temperature phase transition at $\Gamma_{c}=J / 2$. The $Z_{2}$ symmetry mentioned after Eq. (11) is spontaneously broken and $\left\langle S_{i}^{z}\right\rangle$ becomes non-zero when one crosses from the paramagnetic phase at $\Gamma>J / 2$ into the ferromagnetic phase $\Gamma<J / 2$.

In the plane of $\left(k_{B} T / J, \Gamma / J\right)$, there is a ferromagnetic (FM) region in which the solution with $m \neq 0$ has a lower free energy (the $Z_{2}$ symmetry is broken), and a paramagnetic (PM) region in which $m=0$. The boundary between the two is obtained by taking the limit $m \rightarrow 0$ in Eq. (5). This gives $2 \Gamma / J=\tanh (\beta \Gamma / 2)$, i.e.,

$$
\frac{k_{B} T}{J}=\frac{\Gamma}{J}\left[\ln \left(\frac{1+2 \Gamma / J}{1-2 \Gamma / J}\right)\right]^{-1} .
$$

The phase diagram is shown in Fig. [1

\section{B. Holstein-Primakoff Approach}

We shall now find the low-energy spectrum of the Hamiltonian in Eq. (2). The form of (2) shows that the total spin $\vec{S}_{t o t}^{2}=S(S+1)$ is a good quantum number; $S$ can take any value from $N / 2$ down to $1 / 2$ or 0 , depending on whether $N$ is odd or even. For each value of $S$, the Hamiltonian describes a single spin; we shall see below that the low energy properties of the system are dominated by values of $S$ lying close to $N / 2$. The multiplicities $D(S)$ for different values of $S$ (not counting the trivial multiplicity of $2 S+1$ due to different values of $\left.S_{\text {tot }}^{z}\right)$ are given by the following expressions: $D(N / 2)={ }^{N} C_{0}=1, D(N / 2-1)={ }^{N} C_{1}-{ }^{N} C_{0}=N-1$, $D(N / 2-2)={ }^{N} C_{2}-{ }^{N} C_{1}=N(N-3) / 2$ and so on. In general, if $p$ is an integer satisfying $3 \leq p \leq N / 2$, then

$$
\begin{aligned}
& D(N / 2-p)={ }^{N} C_{p}-{ }^{N} C_{p-1} \\
& =\frac{N(N-1) \cdots(N-p+2)}{p !}(N-2 p+1) .
\end{aligned}
$$




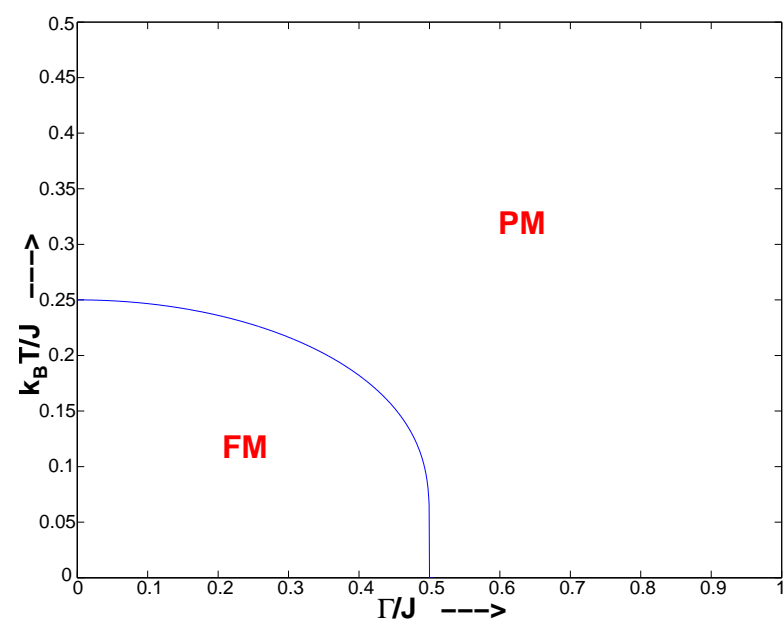

FIG. 1: Phase diagram of the model in mean field theory. FM and PM denote ferromagnetic and paramagnetic regions respectively.

For $N \rightarrow \infty$ and $p<<N / 2$, the leading term in $D(N / 2-$ $p)$ is given by $N^{p} / p !$.

We shall now find the ground state and low-lying excitations of Eq. (2) in a sector with a given value of the total spin $S$, assuming that $\Gamma>J / 2$. To do this, we use the Holstein-Primakoff transformation,

$$
\begin{aligned}
S_{t o t}^{x} & =S-a^{\dagger} a, \\
S_{t o t}^{z}-i S_{t o t}^{y} & =\sqrt{2 S-a^{\dagger} a} a, \\
S_{t o t}^{z}+i S_{t o t}^{y} & =a^{\dagger} \sqrt{2 S-a^{\dagger} a},
\end{aligned}
$$

where $\left[a, a^{\dagger}\right]=1$. Assuming that $S$ is close to $N / 2$ which is a large number, we make the approximation of ignoring $a^{\dagger} a$ with respect to $2 S$ in the last two equations in (8); this gives

$$
\begin{aligned}
S_{t o t}^{x} & =S-a^{\dagger} a, \\
S_{t o t}^{z} & =\sqrt{\frac{S}{2}}\left(a+a^{\dagger}\right) .
\end{aligned}
$$

The Hamiltonian in Eq. (2) then takes the form

$$
H=-\Gamma S+\Gamma a^{\dagger} a-\frac{J S}{4 N}\left(a^{\dagger}+a\right)^{2} .
$$

The spectrum of this Hamiltonian can be found by transforming to the variables $q$ and $p$, where

$$
a=\frac{q+i p}{\sqrt{2}}, \text { and } a^{\dagger}=\frac{q-i p}{\sqrt{2}},
$$

and $[q, p]=i$. Then Eq. (10) takes the form

$$
H=-\Gamma\left(S+\frac{1}{2}\right)+\frac{1}{2} \Gamma p^{2}+\frac{1}{2}\left(\Gamma-\frac{J S}{N}\right) q^{2} .
$$

This describes a simple harmonic oscillator with the frequency

$$
\omega_{S}=\Gamma \sqrt{1-\frac{J S}{\Gamma N}}
$$

[Since the maximum value of $S$ is $N / 2$, we see that $\omega_{S}$ is real since $\Gamma>J / 2$. If $\Gamma<J / 2$, we have to analyze the problem differently as discussed below.] The energy spectrum of Eq. (12) is given by

$$
E_{S, n}=-\Gamma\left(S+\frac{1}{2}\right)+\left(n+\frac{1}{2}\right) \omega_{S},
$$

where $n=0,1,2, \cdots$. The ground state of the system corresponds to $S=N / 2$ and $n=0$.

Since $S$ takes the values $N / 2, N / 2-1, N / 2-2, \cdots$, Eq. (14) shows that there are two towers of equally spaced excitations: one with a spacing of $\Gamma$ coming from the first term (arising from excitations in which the total spin changes) and the other with a spacing $\omega_{S}$ coming from the second term (arising from excitations in which the total spin does not change). Only the energy spacing $\Gamma$ turns out to be thermodynamically significant; this is due to the multiplicities given in Eq. (17) as we will now see. The partition function of the oscillator in Eq. (14) at an inverse temperature $\beta$ is given by

$$
Z(S)=\frac{e^{\beta \Gamma(S+1 / 2)}}{2 \sinh \left(\beta \omega_{S} / 2\right)} .
$$

The complete partition function is therefore given by

$$
Z=\sum_{S} D(S) Z(S)
$$

In the thermodynamic limit $N \rightarrow \infty$, the sum in Eq. (16) will be dominated by values of $S$ lying close to $N / 2$. We can therefore write the partition function as

$$
Z \simeq \sum_{p=0}^{\infty} \frac{N^{p}}{p !} \frac{e^{\beta \Gamma(N / 2-p+1 / 2)}}{2 \sinh (\beta \omega / 2)},
$$

where we have approximated $\omega$ by its value at $S=N / 2$, namely, $\omega \simeq \Gamma \sqrt{1-J / 2 \Gamma}$. The free energy per spin is then given by

$$
\begin{aligned}
f & =-\lim _{N \rightarrow \infty} \frac{\log Z}{N \beta} \\
& =-\frac{\Gamma}{2}-\frac{e^{-\beta \Gamma}}{\beta},
\end{aligned}
$$

to lowest order in the quantity $e^{-\beta \Gamma}$, in the limit of zero temperature. This shows that the thermodynamic gap is given by $\Gamma$. Note that this agrees with the gap obtained in mean field theory. The harmonic oscillator energy levels with spacing $\omega_{S}$ correspond to a collective excitation of all the spins and therefore they do not appear in mean field theory (which only takes into account excitations at a single site).

We will end with a brief discussion of the case $\Gamma<$ $J / 2$. A classical analysis with $\mathbf{S}=\left(S^{x}, S^{y}, S_{\text {tot }}^{z}\right)=$ $S(\cos \phi \sin \theta, \sin \phi \sin \theta, \cos \theta)$, where $\theta$ and $\phi$ are the usual polar and azimuthal angles, gives the Hamiltonian

$$
\mathcal{H}[\theta, \phi]=-\frac{J S^{2}}{2 N} \cos ^{2} \theta-\Gamma S \sin \theta \cos \phi .
$$


The lowest energy configuration of this is given by $S=$ $N / 2, \theta=\sin ^{-1}(2 \Gamma / J)$ and $\phi=0$. There are two solutions for $\theta$, one lying in the range $[0, \pi / 2]$ and the other in the range $[\pi / 2, \pi]$. We choose either one of these as the ground state and perform a Holstein-Primakoff transformation around it. We then find that there are again two towers of equally spaced excitations, one with spacing $J / 2$ and the other with spacing $(J / 2) \sqrt{1-4 \Gamma^{2} / J^{2}}$ corresponding to a collective excitation. The thermodynamically significant spacing is $J / 2$.

Note that as $\Gamma$ approaches $J / 2$ from either above or below, the collective excitation energy softens and goes to zero. Thus it is the collective excitation gap (not the thermodynamically significant gap) which is sensitive to the QCP lying at $\Gamma_{c}=J / 2$.

We would like to emphasize here that our model has infinite range interactions and we are interested in the thermodynamic limit $N \rightarrow \infty$. In that limit, the effective Hamiltonian seen by any one spin is just the mean field produced by all the other spins. Hence there is no difference between the phase diagrams obtained by the mean field theory and the Holstein-Primakoff approach.

A difference between mean field theory and the Holstein-Primakoff approach is that only the latter correctly describes the collective excitation which involves all the spins (as we have discussed above). However, a single mode like the collective excitation does not play any role in determining the phase diagram in the thermodynamic limit.

\section{QUENCH DYNAMICS}

In this section, we will study the quench dynamics of the spin model across the QCP. We saw above that it is the collective excitations (in which the total spin does not change) which are sensitive to the presence of a QCP. We will therefore restrict our attention to the lowest energy sector in which the total spin is given by $S=N / 2$.

A quench dynamics across a QCP has been studied earlier for Ising-like systems with short-range interactions ${ }^{5}$ and driven XY spin chains ${ }^{6}$. Here, taking advantage of the fact that the large $S$ (and hence classical) limit of our model is also the thermodynamic limit, we shall derive an analytical expression for the long-time average of the EOC in the thermodynamic limit as the system is quenched across the QCP starting from the paramagnetic phase. In this section, we shall mostly follow the method of Ref. 5 .

\section{A. Quantum Analysis}

To begin with, we study the dynamics of the EOC (defined as $\left.\left\langle\left(S_{t o t}^{z}\right)^{2}\right\rangle / S^{2}\right)$ by changing the transverse field $\Gamma$ from an initial value $\Gamma_{i} \gg \Gamma_{c}$ to a final value $\Gamma_{f}$ suddenly, so that the ground state of the system has no time to change during the quench. In this case, just after the quench, the ground state of the system can be expressed, in terms of the eigenstates $|n\rangle$ of the new Hamiltonian $\mathcal{H}_{f}=-(J / 4 S)\left(S_{\text {tot }}^{z}\right)^{2}-\Gamma_{f} S_{\text {tot }}^{x}$ as

$$
|\psi\rangle=\sum_{n} c_{n}|n\rangle
$$

where $c_{n}$ denotes the overlap of the eigenstate $|n\rangle$ with the old ground state $|\psi\rangle$. As the state of the system evolves, it is given at time $t$ by

$$
|\psi(t)\rangle=\sum_{n} c_{n} e^{-i E_{n} t / \hbar}|n\rangle,
$$

where $E_{n}=\left\langle n\left|\mathcal{H}_{f}\right| n\right\rangle$ are the energy eigenvalues of the Hamiltonian $\mathcal{H}_{f}$. The EOC can thus be written as

$$
\begin{array}{r}
\left\langle\psi(t)\left|\left(S_{\text {tot }}^{z}\right)^{2} / S^{2}\right| \psi(t)\right\rangle=\sum_{m, n} c_{n} c_{m} \cos \left[\left(E_{n}-E_{m}\right) t / \hbar\right] \\
\times\left\langle m\left|\left(S_{\text {tot }}^{z}\right)^{2} / S^{2}\right| n\right\rangle \cdot(22)
\end{array}
$$

Eq. (22) can be solved numerically to obtain the time evolution of the EOC. However, certain qualitative features of the dynamics can be extracted from Eq. (22) without resorting to numerics. First, we note that when $\Gamma_{f}$ and $\Gamma_{i}$ both lie in the paramagnetic phase, we expect the old ground state to have a large overlap with the new one, so that $c_{n} \simeq \delta_{n 1}$. In this case, $\left\langle\left(S_{\text {tot }}^{z}\right)^{2}(t)\right\rangle$ is expected to undergo small oscillations about $\left\langle\left(S_{t o t}^{z}\right)^{2}(t=0)\right\rangle$. On the other hand, if $\Gamma_{f} \ll \Gamma_{c} \ll \Gamma_{i}$, there is very little overlap between the two ground states and the amplitude of oscillation is again expected to be small. Note that the situation is completely different from the adiabatic turning on of $\Gamma$, where for $\Gamma_{f} \ll \Gamma_{c}$, the system has a maximal value of $\left\langle\left(S_{t o t}^{z}\right)^{2}\right\rangle$. In between these two regimes, when $\Gamma_{f} \simeq \Gamma_{c}$, the old ground state is expected to have significant overlap with many eigenstates $|m\rangle$, and the oscillation amplitude can be expected to be large. These qualitative expectations are verified in Fig. 22 Here, we have quenched the transverse fields to $\Gamma_{f} / J=0.9,0.01$, and 0.4 starting from $\Gamma_{i} / J=2.0$. The oscillation amplitudes of the EOC for $S=100$, as shown in Fig. 22 are small for $\Gamma_{f}=0.9$ and 0.01 , whereas it is substantially larger for $\Gamma_{f}=0.4$.

Next, to understand the dynamics of the EOC in a little more detail, we study its long-time averaged value given by

$$
\begin{aligned}
O & =\lim _{T \rightarrow \infty}\left\langle\left\langle\left(S_{\text {tot }}^{z}\right)^{2}(t)\right\rangle\right\rangle_{T} / S^{2} \\
& =\frac{1}{S^{2}} \sum_{n} c_{n}^{2}\left\langle n\left|\left(S_{t o t}^{z}\right)^{2}\right| n\right\rangle
\end{aligned}
$$

for different $\Gamma_{f}$. Note that the long-time average depends on the product of the overlap of the state $|n\rangle$ with the old ground state and the expectation of $\left(S_{t o t}^{z}\right)^{2}$ in that state. From our earlier discussion regarding the dynamics of $\left\langle\left(S_{t o t}^{z}\right)^{2}\right\rangle$, we therefore expect $O$ to have a peak somewhere near the critical point on the ordered side where 


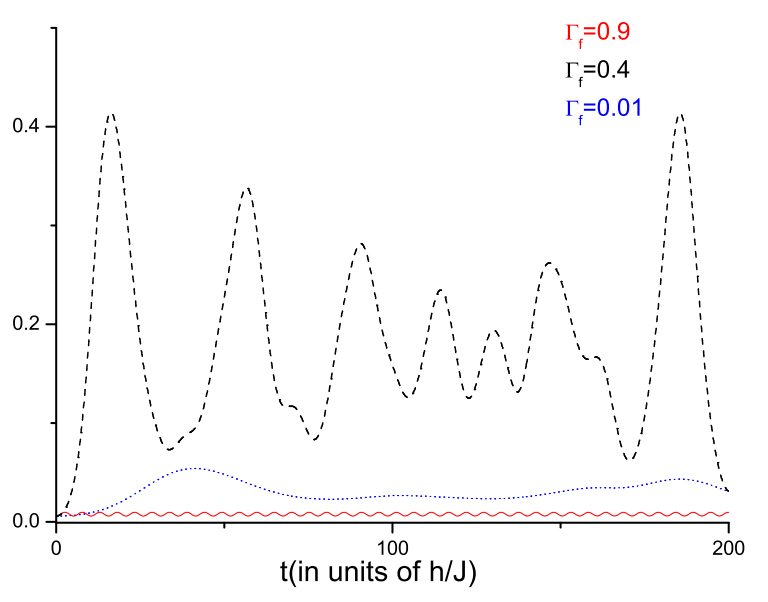

FIG. 2: Dynamics of $\left\langle\left(S_{\text {tot }}^{z}\right)^{2}\right\rangle / S^{2}$ for $S=100$ after quenching the transverse field to different values $\Gamma_{f} / J$ from an initial field $\Gamma_{i} / J=2$. The oscillation amplitudes are small, as seen from the solid (red) and dotted (blue) curves corresponding to $\Gamma_{f} / J=0.9$ and 0.01 respectively, far away from the critical point $\Gamma_{c} / J=0.5$. The oscillation is large in the ordered phase near the critical point as seen from the dashed (black) curve $\Gamma_{f} / J=0.4$.

such an overlap is maximized. This is verified by explicit numerical computation of Eq. (23) in Fig. 3 for several values of $S$ and $\Gamma_{i} / J=2$. We find that $O$ peaks around $\Gamma_{f} / J=0.25$, and the peak height decreases slowly with increasing $S$.

\section{B. Classical Analysis}

To understand the position and the system size dependence of the peak in $O$, we now look at the thermodynamic (large system size) limit; in the present model, this is also the large $S$ and therefore classical limit. With this observation, we study the classical equations of motion for $\mathbf{S}=S(\cos \phi \sin \theta, \sin \phi \sin \theta, \cos \theta)$ for $\Gamma=\Gamma_{f}$. In the present model, $S$ is a constant. Thus in the classical limit, we need to study the equations of motion for $\theta$ and $\phi$. To this end, we note that the classical Lagrangian can be written in terms of $\theta$ and $\phi$ as $\frac{13}{3}$

$$
L=-S[1-\cos \theta] \frac{d \phi}{d t}-\mathcal{H}[\theta, \phi],
$$

This gives the equations of motions

$$
\begin{aligned}
\frac{d \theta}{d t} & =\Gamma_{f} \sin \phi \\
\frac{d \phi}{d t} & =-\frac{J}{2} \cos \theta+\Gamma_{f} \cot \theta \cos \phi
\end{aligned}
$$

Eq. (25) has to be supplemented with the initial condition that $S_{t o t}^{x}=S$ at $t=0$. The condition $S_{\text {tot }}^{x}=S$

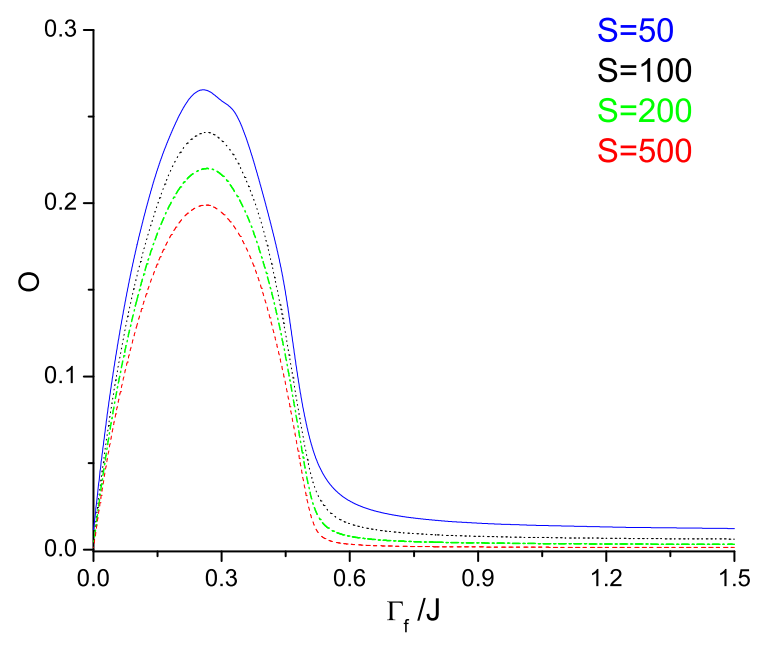

FIG. 3: Plot of the long-time average $O$ as a function of $\Gamma_{f} / J$ for different $S$. In the plot the solid (blue), dotted (black), dash-dotted (green) and the dashed (red) lines represents respectively the results for $S=50, S=100, S=200$ and $S=500$. $O$ peaks around $\Gamma_{f} / J=0.25$ and the peak value decreases with increasing $S$. For all plots we have chosen $\Gamma_{i} / J=2$.

corresponds to $\theta=\pi / 2, \phi=0$ which is also a fixed point of Eq. (25). Therefore we shall start from an initial condition which is very close to the fixed point: $\theta=\pi / 2-\epsilon, \phi=\epsilon$, where $\epsilon$ is an arbitrarily small constant. Further, since the motion occurs on a constant energy surface after the quench has taken place, we have

$$
\Gamma_{f}=\frac{J}{4} \cos ^{2} \theta+\Gamma_{f} \sin \theta \cos \phi .
$$

Using Eqs. (25) and (26), we get an equation of motion for $\theta$ in closed form,

$$
\frac{d \theta}{d t}=\frac{\sqrt{\Gamma_{f}^{2} \sin ^{2}(\theta)-\left[\Gamma_{f}-\frac{J}{4} \cos ^{2} \theta\right]^{2}}}{\sin \theta} \equiv f(\theta) .
$$

It can be seen that the motion of $\theta$ is oscillatory and has classical turning points at $\theta_{1}=\sin ^{-1}\left(\left|1-4 \Gamma_{f} / J\right|\right)$ and $\theta_{2}=\pi / 2$. One can now obtain $\left\langle\left(S_{\text {tot }}^{z}\right)^{2}\right\rangle_{T}=\left\langle\cos ^{2} \theta\right\rangle_{T}$ from Eq. (27),

$$
\begin{aligned}
\left\langle\cos ^{2} \theta\right\rangle_{T} & =\mathcal{N} / \mathcal{D}, \\
\mathcal{N} & =\int_{\theta_{1}}^{\theta_{2}} d \theta \frac{\cos ^{2} \theta}{f(\theta)} \\
& =4 \sqrt{8 \Gamma_{f}\left(J-2 \Gamma_{f}\right)} / J \\
\mathcal{D} & =\int_{\theta_{1}}^{\theta_{2}} d \theta \frac{1}{f(\theta)} .
\end{aligned}
$$

When trying to evaluate $\mathcal{D}$, we find that the integral has an end-point singularity at $\theta_{2}$; this can be regulated by a 
cut-off $\eta$ so that $\theta_{2}=\pi / 2-\eta$. With this regularization, $\mathcal{D}=-J \ln (\eta) / \sqrt{\Gamma_{f}\left(J-2 \Gamma_{f}\right) / 2}$. The cut-off used here has a physical meaning and is not arbitrary. To see this, note that the angles $(\theta, \phi)$ define the surface of a unit sphere of area $4 \pi$. This surface, for a system with spin $S$, is also the phase space which has $2 S+1$ quantum mechanical states. For large $S$, the area of the surface occupied by each quantum mechanical state is therefore $4 \pi /(2 S+1) \simeq 2 \pi / S$. In other words, each quantum mechanical state will have a linear dimension of order $1 / \sqrt{S}$; this is how close we can get to a given point on the surface of the sphere. Note that this closeness is determined purely by quantum fluctuation and vanishes for $S \rightarrow \infty$. Thus $\eta$, which is also a measure of how close to the point $\theta=\pi / 2$ we can get, must be of the order of $1 / \sqrt{S}$; this determines the system-size dependence of $\left\langle\cos ^{2} \theta\right\rangle_{T}$. Using Eq. (28), we finally get

$$
\left\langle\cos ^{2} \theta\right\rangle_{T}=\frac{16 \Gamma_{f}\left(J-2 \Gamma_{f}\right)}{J^{2} \ln (S)} .
$$

Eq. (31) is one of the main results of this work. It demonstrates that the long-time average of the EOC must be peaked at $\Gamma_{f} / J=0.25$ which agrees perfectly with the exact quantum mechanical numerical analysis leading to Fig. 3] Moreover, it provides an analytical understanding of the $S$ (and hence system-size) dependence of the peak values of $\Gamma_{f} / J$. A plot of the peak height of $O$ as a function of $1 / \ln (S)$ indeed fits a straight line, as shown in Fig. 4. So we conclude that the peak in $O$ vanishes logarithmically with system size $S$.

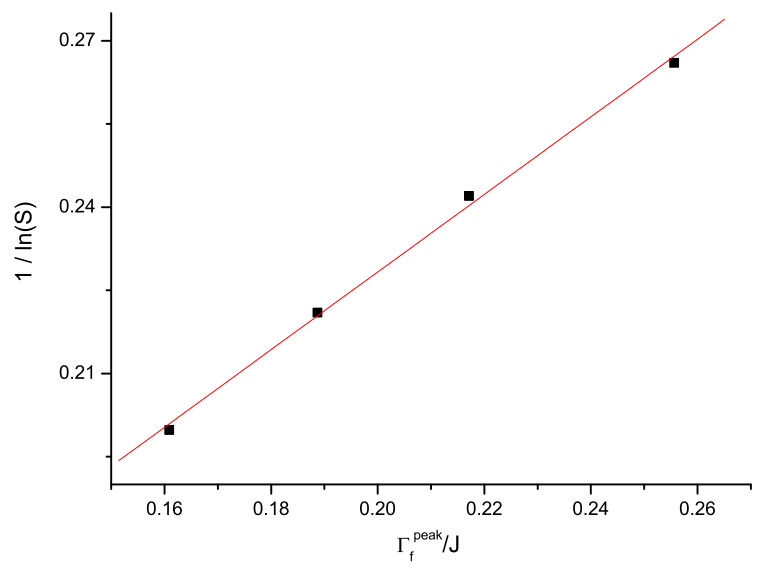

FIG. 4: Plot of the maximum peak height $O_{\max }$ of the longtime average of the EOC as a function of $1 / \ln (S)$. The straight line shows the linear fit.

\section{AC DYNAMICS}

In this section we study the dynamics of the system in the presence of an oscillatory transverse field. As in

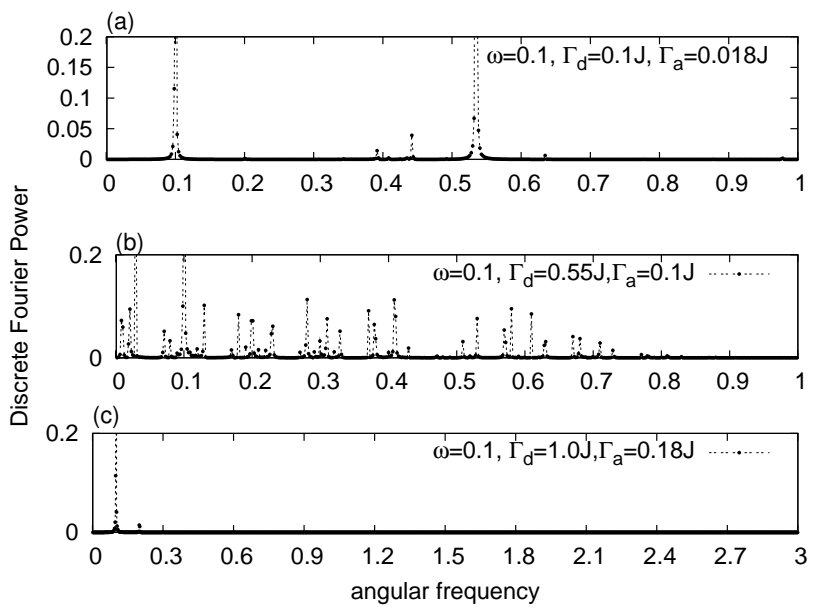

FIG. 5: Plot of the DFT power spectrum of the EOC $\left\langle\psi(t)\left|\left(S_{t o t}^{z}\right)^{2}\right| \psi(t)\right\rangle / S^{2}$ for different values of $\Gamma_{d}$. Panels (a) and (c) show the DFTs in the ferromagnetic and paramagnetic phases respectively, whereas panel (b) shows the same for the quantum critical region. In all three panels, the height of the peak at the drive frequency $(\omega=0.1)$ is maximum and is scaled to 1 (chopped off in the figure). The plots clearly indicate contributions from a relatively large number of frequencies to the DFT power spectrum near the QCP.

Sec. IIII we shall first study the quantum dynamics of the system in the presence of a transverse ac field. This will be followed by an analysis of the classical equations of motion (Eq. (25)) which describe the behavior of the system in the large $S$ limit.

\section{A. Quantum Analysis}

In the presence of a transverse $\mathrm{AC}$ field, the total Hamiltonian is

$$
H(t)=-\frac{J}{2 N}\left(S_{t o t}^{z}\right)^{2}-\left[\Gamma_{d}+\Gamma_{a} \cos (\omega t)\right] S_{t o t}^{x},
$$

where $\Gamma_{d}$ denotes the static part of the transverse field, $\Gamma_{a}$ is the amplitude of the $\mathrm{AC}$ part, and $\omega$ is the drive frequency. Note that for small $\Gamma_{a}, \Gamma_{d}$ determines the point in the phase diagram about which the AC dynamics takes place. For the rest of this work we shall restrict ourselves to this limit and for definiteness take a small ratio $\Gamma_{a} / \Gamma_{d}=0.18$. We have checked that the qualitative picture of the $\mathrm{AC}$ dynamics that we describe below remains the same for a large range of this ratio as long as $\Gamma_{a} / \Gamma_{d} \ll 1$.

To elucidate the properties of the AC dynamics of our model, we calculate the EOC $\left\langle\psi(t)\left|\left(S_{t o t}^{z}\right)^{2}\right| \psi(t)\right\rangle / S^{2}$. For a sufficiently small driving frequency $\omega$, we are in the adiabatic regime and the system always stays in the instantaneous ground state of $H(t)$. In this case, the behavior of the EOC is the same as that for the static case, and has been well studied ${ }^{2}$. However, the situation changes when we drive the system with moderately high frequen- 
cies due to possible Landau-Zener type transitions between different time-dependent levels of $H(t)$, and a full out-of-equilibrium analysis of the problem becomes necessary. If, however, the driving frequency becomes very high compared to other natural frequency scales of the system, the eigenstates of $H(t)$ vary much faster compared to the characteristic time scale of most of the possible Landau-Zener transitions. Hence such transitions fail to take place and the response is characterized by a few trivial frequencies. In this work, we shall therefore investigate the case of moderately high frequency and study the characteristics of the frequency spectrum of the EOC. For the sake of definiteness, we shall choose $\omega=0.1 J$ in the rest of this work.

For a given set of parameters $\left(\Gamma_{d}, \Gamma_{a}\right.$ and $\left.\omega\right)$, we start with the ground state of $H(t=0)$ (in Eq. (32) ) as the initial state $|\psi(t=0)\rangle$. We then solve the time-dependent Schrödinger equation to obtain the time evolution of the state $|\psi(t)\rangle$, using 4th order Runge-Kutta method with adaptive step-size contro ${ }^{14}$. The time variation of the EOC is found, and the discrete Fourier transform (DFT) of the data is taken to obtain its frequency spectrum.
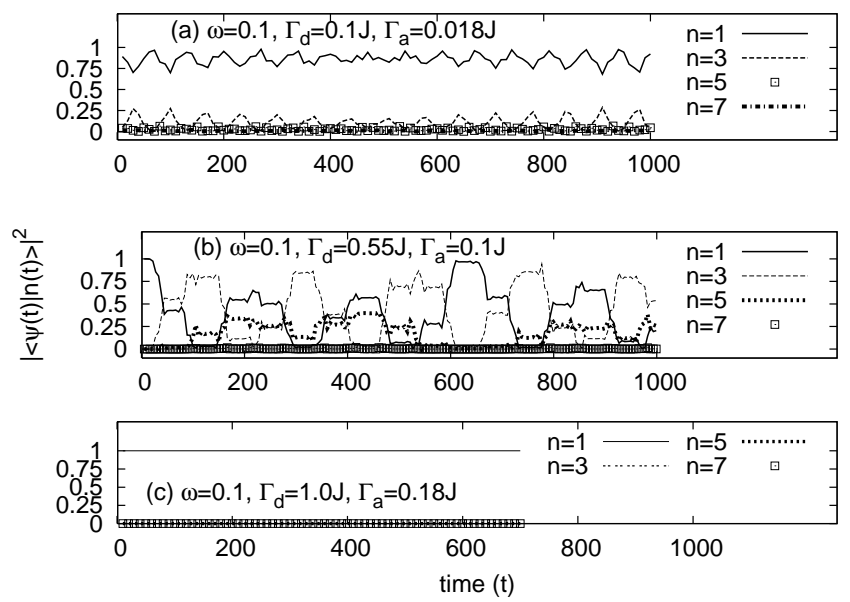

FIG. 6: The time evolution (in units of $\hbar / J$ ) of the overlaps of the instantaneous state $|\psi(t)\rangle$ with the time-dependent eigenstates $|n(t)\rangle$ of the total Hamiltonian $H(t)(n=1$ denotes the ground state, $n=2$ the first excited state and so on). Results are shown for $n=1,3,5$ and 7 .

The DFT power spectra show that inside the paramagnetic and ferromagnetic phases, the response of the system is dominated by a few frequencies (panels (a) and (c) of Fig. 51). These are either the driving frequency $\omega=0.1 J$ or the natural oscillation frequencies of the system as will be shown in more details in Sec. IVB In contrast, near the QCP (panel (b) of Fig. [5), several frequencies appear in the spectrum. Thus we find that the EOC displays a noisy behavior in the time domain near the QPT.

To look into the origin of these frequencies, we have plotted the instantaneous overlaps of the time dependent state $|\psi(t)\rangle$ with the first few instantaneous eigenstates $|n(t)\rangle$ 's of $H(t)$, for $\omega=0.1$ in Fig. [6] These overlaps are significant only for $n=1$ (ground state), $n=3,5$ and 7 for all times. The overlap is negligible for all other odd $n$ and vanishes for all even $n$ by symmetry. (The states with odd and even values of $n$ have opposite values of the $Z_{2}$ parity mentioned after Eq. (11). Hence the matrix element of $S_{t o t}^{x}$ between states with odd and even $n$ vanishes). In addition, we have also plotted the instantaneous gaps $G_{m n}(t)=E_{n}(t)-E_{m}(t)$ in Fig. 17. In the ferromagnetic region, as shown in panel (a) of Fig. 6. the transitions are mainly between the two levels, $n=1$ and 3. However, the overlaps have much higher average value for $n=1$ than that for $n=3$. This indicates that the system mostly stays close to the ground state. Also, as can be seen from panel (a) of Fig. 7 the relevant gaps $G_{m n}(t)$ are almost constant, resulting in a periodic behavior dominated by the driving frequency $\omega=0.1 \mathrm{~J}$ and a frequency $0.52 J$ which is close to the almost static value of the gaps. Deep inside the paramagnetic region (panel (c) of Fig. 6), the system is found to be in the ground state $(|n=1(t)\rangle)$ throughout the evolution; all other overlaps tend to zero. This is because in this phase, as can be seen from panel (c) of Fig 7 the instantaneous gaps $G_{m n}(t)=E_{n}(t)-E_{m}(t)$ always remain large and prohibits occurrence of any transition from the ground state to the higher levels. Thus the dynamics is again governed by a few frequencies and has a simple periodic behavior. In contrast, near the QCP (panel (b) of Fig. 6) all of the levels $n=1,3$ and 5 have substantial overlaps with the instantaneous ground state ( $n=7$ also has a small contribution). The overlaps are much flatter and more evenly distributed among the levels $n=1$ and 3 on an average. Further, as can be seen from panel (b) of Fig. 7 the gaps $G_{m n}$ undergo appreciable oscillations, creating possibilities of Landau-Zener transitions at multiple frequencies. Thus, quite generally, the AC dynamics near the QCP involves multiple frequencies and is expected to exhibit noisy behavior in the time domain.

Before ending this section, we would like to point out that the time evolution of $\left\langle\left(S_{t o t}^{z}\right)^{2}\right\rangle$, for some specific parameter values, may have large oscillations with an unusually long time period and discuss the reason for its occurrence. To this end, we present an example of this phenomenon in Fig. 8 The figure shows oscillations with a time period of about 1100 which is much longer than the period of the oscillating transverse field, namely, $2 \pi / \omega \simeq 63$. This phenomenon is well-known in the context of Rabi oscillations which can occur when a two-state system is subjected to a periodic potential ${ }^{15}$. For instance, consider a Hamiltonian which is a $2 \times 2$ matrix of the form $\left(\omega_{0} / 2\right) \sigma^{z}+(b \cos \omega t) \sigma^{x}$. If $b$ is small and we start initially in the ground state of the term $\left(\omega_{0} / 2\right) \sigma^{z}$ (the energy gap corresponding to this term is given by $\left.\omega_{0}\right)$, we find that if $\omega$ is close to $\omega_{0}$, the system periodically makes transitions to the excited state and returns to the ground state. The time period of this oscillation is given by $2 \pi / \sqrt{\left(\omega-\omega_{0}\right)^{2}+b^{2}}$; this is much longer than $2 \pi / \omega$ if $\omega / \omega_{0}$ is close to 1 and $b \ll \omega$. The amplitude 

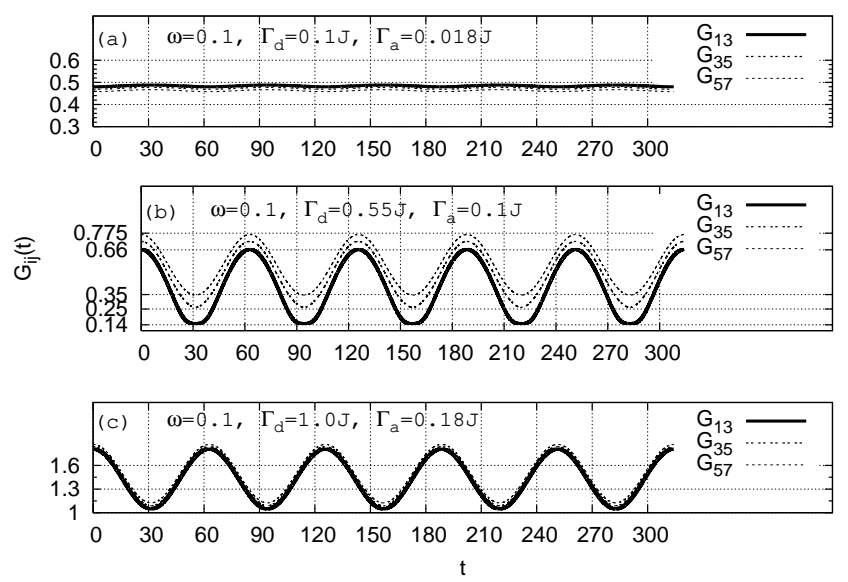

FIG. 7: Plot of the relevant gaps $G_{m n}(t)=E_{n}(t)-E_{m}(t)$ between different eigenvalues of $H(t)$ as a function of time (in units of $\hbar / J$ ) for different values of $\Gamma_{d}$. In (a), in the ferromagnetic phase $\left(\Gamma_{d}=0.1\right)$, the gaps are almost static and equal at all times. In (b), near criticality $\left(\Gamma_{d}=0.55\right)$, the gaps vary considerably with time and attain quite low stationary values. In (c), in the paramagnetic phase $\left(\Gamma_{d}=1.0\right)$, the gaps vary considerably with time, but are always much larger than their counterparts in the other two phases.

of the oscillations is maximum for $\omega=\omega_{0}$. (Similar long time period oscillations can occur if $\omega / \omega_{0}$ is close to $1 / 2,1 / 3, \cdots)$. Returning to our problem, for the parameters given in Fig. 8 we find that the system is quite well described if we only keep the first three eigenstates corresponding to $n=1,3,5$ of the time-independent part of the Hamiltonian in Eq. (32). If we truncate the Hamiltonian to only these three states and study the AC dynamics starting initially with the first eigenstate, we find that the probabilities of being in the three states have large and long time period oscillations as shown in Fig. 9 We thus have a three-state version of Rabi oscillations. [One of the parameters used in Fig. 8] $\Gamma_{a}$, has been chosen to be different compared to the earlier calculations. This is because the presence of Rabi oscillations is sensitively dependent on the parameter values; it was necessary to change the value of $\Gamma_{a}$, keeping the other parameters fixed, to show a striking example of such oscillations.] Note that although the probability of being in the state $n=5$ is small at all times as shown in Fig. 9] it is necessary to keep this state in order to obtain the longtime oscillations. We have checked numerically that if we truncate the Hamiltonian to only the states $n=1$ and 3 , we do not get these large and long-time oscillations. We also note that the driving frequency $\omega=0.1$ is close to one-fourth of the gap between the states $n=1$ and 3 , namely, $G_{13}=0.408$. The Rabi oscillations are found to peak at $\omega \simeq 0.101$ and to disappear if $\omega$ is changed from that value by just $4 \%$. Therefore it is expected that the low-frequency peaks in the DFT spectrum are not generic features at all drive frequencies, but will be seen only for special values of the drive frequencies.
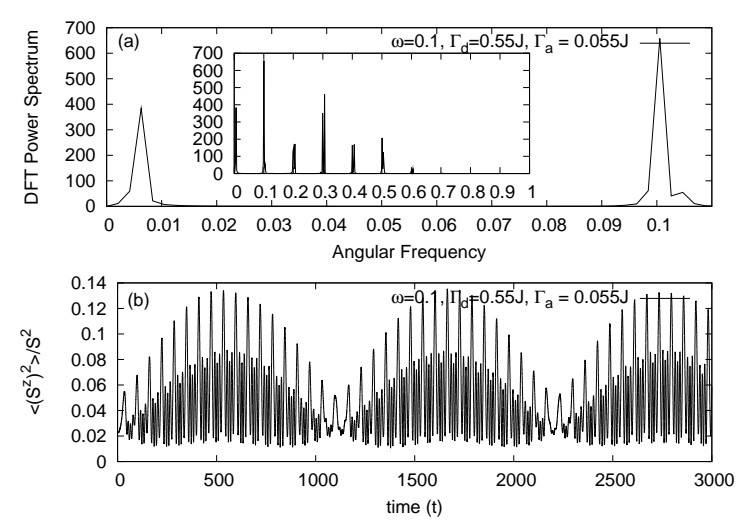

FIG. 8: Plot of the DFT power spectrum in panel (a) and the time evolution in panel (b) of the EOC $\left\langle\psi(t)\left|\left(S_{t o t}^{z}\right)^{2}\right| \psi(t)\right\rangle / S^{2}$ for $S=50, \Gamma_{d}=0.55, \Gamma_{a}=0.055$, and $\omega=0.1$. Note the oscillations with a very large time period of about 1100 in panel (b), and the corresponding peak at a frequency of about 0.006 in panel (a).

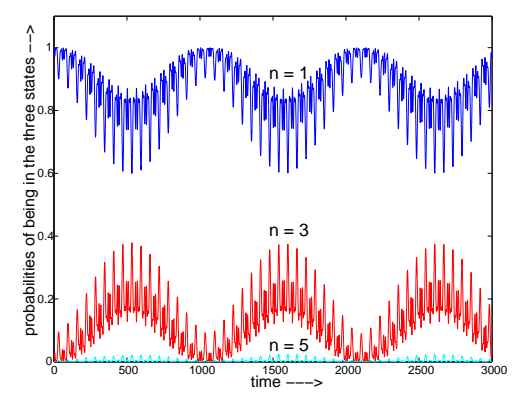

FIG. 9: Time evolution of the probabilities of being in different states when the model considered in Fig. 8 is truncated to the three states $n=1,3$ and 5 .

\section{B. Classical Analysis}

The classical analysis of the AC dynamics of the model involves a numerical solution of Eq. (25) with $\Gamma_{f} \rightarrow$ $\Gamma_{d}+\Gamma_{a} \cos (\omega t)$. As in the case of the quantum analysis, we are going to restrict our analysis to an intermediate drive frequency $\omega=0.1 J$ and $\Gamma_{d} \gg \Gamma_{a}$. Note that we are interested in the classical limit of the quantum dynamics, for which the system is in it's ground state at $t=0$. Thus we choose the initial condition for the classical dynamics such that the system is close to its fixed point. These fixed points, as can be easily seen from Eq. (25), are given by $\left(\theta_{f}, \phi_{f}\right)=(\pi / 2,0)$ when the system is in the paramagnetic phase with $\Gamma_{d} \geq 0.5$, and by $\left(\theta_{f}, \phi_{f}\right)=$ $\left(\sin ^{-1}\left(2 \Gamma_{d}\right), 0\right)$ for the ferromagnetic phase where $\Gamma_{d}<$ 0.5. Note that the fixed point structure of Eq. (25) changes at $\Gamma_{d} / J=0.5$ which is also the location of the QCP. Therefore, we choose the initial condition $\left(\theta_{f}-\epsilon, \epsilon\right)$ for small $\epsilon=0.1$. We have checked that smaller values of $\epsilon$ do not lead to qualitative change in the essential features of the dynamics. Also, in the rest of this section, 
we shall maintain the same ratio $\Gamma_{a} / \Gamma_{d}=0.18$ as in the previous section.

Before carrying out the numerical simulations, it is useful to obtain a qualitative understanding of the dynamics of the system in the presence of the driving field. Since the amplitude of the drive field $\Gamma_{a}$ is small and the motion starts from near the classical fixed point, we expect that a small amplitude analysis for the motion of $\theta$ and $\phi$ would give us a qualitative understanding of the dynamics for both the paramagnetic and ferromagnetic phases. Of course, this expectation is not met near the quantum critical point, and we are going to discuss this point in more detail subsequently.
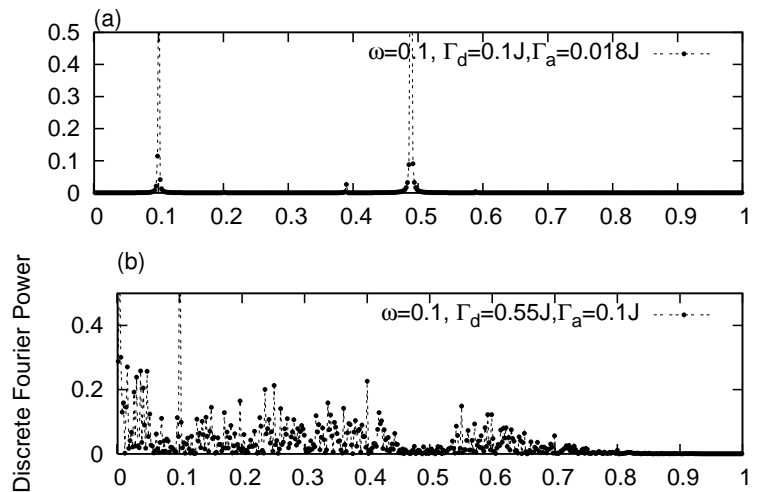

(c)

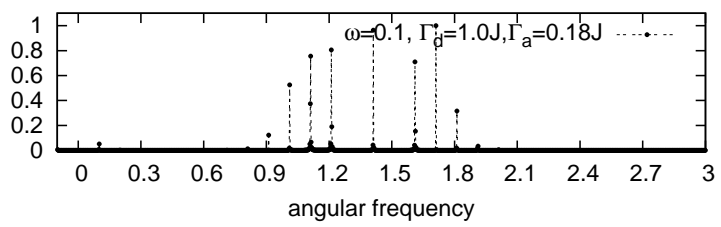

FIG. 10: Plot of the DFT power spectrum of the classical EOC $\cos ^{2} \theta$ for different values of $\Gamma_{d}$, obtained by solving the classical equations of motion in Eq. 25). Panels (a) and (c) show the DFTs in the ferromagnetic and paramagnetic phases respectively; panel (b) shows the same for the critical region. Note that the involvement of many frequencies in the dynamics near the critical point survives even in the classical limit.

Let us first consider the paramagnetic phase where $\Gamma_{d}>J / 2$ and the fixed point is given by $\left(\theta_{f}, \phi_{f}\right)=$ $(\pi / 2,0)$. Linearizing Eqs. (25) about this fixed point, one gets a natural frequency of $\omega_{n}=\sqrt{\Gamma_{f}\left(\Gamma_{f}-J / 2\right)}$ for small amplitude motion. Note that this analysis is only approximate since for our case $\Gamma_{f}$ itself varies in time. But for a small drive amplitude and frequency, where $\Gamma_{d}-\Gamma_{a}=0.8 J \leq \Gamma_{f} \leq \Gamma_{d}+\Gamma_{a}=1.2 J$, we expect the main contribution to the periodic motion of $\theta$ to come from approximately the range $0.49 J \leq \omega_{n} \leq 0.91 J$. Thus the power spectrum for the motion of $\left(S_{t o t}^{z}\right)^{2}(t) / S^{2}=$ $\cos ^{2} \theta$, which has half the time period as $\cos \theta$ in the paramagnetic phase (since $\cos \theta$ runs over both positive and negative values), is expected to have significant contribution from frequencies in the range $0.98 J \leq \omega_{n} \leq 1.8 \mathrm{~J}$ and from the drive frequency $\omega=0.1 J$. This expectation can be verified from panel (c) of Fig. 10 The exact location of all the frequencies, however, cannot be easily deduced from this qualitative argument. Next, let us consider the ferromagnetic phase at $\Gamma_{d}=0.1$. Here the fixed point is given by $\left(\theta_{f}, \phi_{f}\right)=\left(\sin ^{-1}\left(2 \Gamma_{f} / J\right), 0\right)$. A similar linearization about the fixed point leads to a natural frequency of $\omega_{n}=\sqrt{\left(J^{2} / 4\right)-\Gamma_{f}^{2}} \simeq 0.5 J$. Note that in this case, owing to the small ratio of $\Gamma_{d} / J$, the variation of $\Gamma_{f}$ does not affect $\omega_{n}$, at least to leading order in $\Gamma_{f} / J$. Thus we expect that the contribution to the DFT would arise from the drive frequency $\omega=0.1 J$ and the natural frequency $\omega_{n}=0.5 \mathrm{~J}$. This explains the double peaked structure of the DFT spectrum in panel (a) of Fig. 10]

Finally, let us consider the classical equations at $\Gamma_{d}=$ $0.55 \mathrm{~J}$. Here the fixed point at $(\pi / 2,0)$ which corresponds to the energy minima of $H$ (Eq. (21)), is quite shallow and even a small transverse drive field is enough to drive the system far away from the fixed point. Therefore we expect the system to traverse a large part of the phase space during its motion even if $\Gamma_{a}$ is small. Consequently, the DFT spectrum of $\cos ^{2} \theta$ is expected to have contributions from a wide range of frequencies which translates to noisy behavior in the time domain. This expectation is verified by numerical computation as can be seen from panel (b) of Fig. 10] Note that this noisy nature of the $\mathrm{AC}$ dynamics of the EOC occurs due to the presence of a shallow energy minima; this in turn is the consequence of the presence of a QCP at $\Gamma_{d} / J=0.5$ where the fixed point structure of Eq. (25) changes. Therefore we expect that such a feature will be generic for QCPs.

\section{DISCUSSION}

The results obtained in this work can be relevant for certain experimental systems. One possible class of systems where these results may be applicable are ones with long range dipole-dipole interactions such as $\mathrm{KH}_{2} \mathrm{PO}_{4}$ or $\mathrm{Dy}\left(\mathrm{C}_{2} \mathrm{H}_{5} \mathrm{SO}_{4}\right)_{3} 9 \mathrm{H}_{2} \mathrm{O}^{2}$ which exhibit order-disorder transitions driven by tunneling fields. However, the effect of finite-range of the interactions on the dynamics needs to be studied carefully before quantitative predictions can be made. We have left this as a subject of future study.

The other class of systems where our results might be applicable are two-component BECs where the interspecies interaction $(U)$ is strong compared to the intraspecies interaction $\left(U^{\prime}\right)$. Such BECs, in the presence of an external RF drive and a fixed chemical potential, can be described by an effective Hamiltonian ${ }^{16}$

$$
H_{\mathrm{eff}}=\chi S_{z}^{2}+\hbar \Omega S_{x}
$$

where the spin operator $S_{z}=\left(b_{\uparrow}^{\dagger} b_{\uparrow}-b_{\downarrow}^{\dagger} b_{\downarrow}\right) / 2$ and $S_{x}=$ $\left(b_{\uparrow}^{\dagger} b_{\downarrow}^{\dagger}+b_{\downarrow}^{\dagger} b_{\uparrow}^{\dagger}\right) / 2$ are the $z$ and $x$ components of the effective spin in terms of the boson operators $b_{\uparrow, \downarrow}, \uparrow$ and $\downarrow$ determines the species or pseudospin index, $\chi=\left(U-U^{\prime}\right)$ 
is the coefficient of the $S_{z}^{2}$ term, and $\Omega$ is the frequency of the external RF field. Note that $U^{\prime}$ can be made arbitrarily strong compared to $U$ by tuning the system near a Feshbach resonance as discussed for ${ }^{41} K-{ }^{87} R b$ system in Ref. 18. Alternatively, such a Hamiltonian (Eq. 33) with $\chi<0$ can also be realized following the method discussed in Refs. 16 17. The quench dynamics described in our work can then be realized by a sudden change of the frequency of the RF pulse. A more complicated $\mathrm{RF}$ pulse shape can also lead to an effective sinusoidal time-dependent $\Omega$ whereby the AC dynamics can also be realized. We also note here there is a vast body of literature on the model described by Eq. 336.19. Most of these works study the entanglement property of the model for $\chi>0$. In this regime, the model (antiferromagnetic infinite-range Ising model in a transverse field) does not have a second-order quantum phase transition and has completely different properties than the ferromagnetic model that we study here.

In conclusion, we have studied the quench and $\mathrm{AC} d y-$ namics of the EOC of an infinite range ferromagnetic Ising model, both classically and quantum mechanically. We have shown that both the quench and the AC dynamics reflect the presence of the quantum critical point of the model. In particular, the $\mathrm{AC}$ dynamics of the $\mathrm{EOC}$, for a small amplitude and moderate frequency transverse $\mathrm{AC}$ field, exhibits a noisy behavior near the quantum critical point which is qualitatively distinct from its periodic behavior away from the critical point in either the paramagnetic or the ferromagnetic phase. As we have shown, this noisy behavior of the $\mathrm{AC}$ dynamics of the EOC near the QCP follows from quite general arguments, and therefore we expect this behavior to qualitatively hold for more realistic models exhibiting QCP. Finally, we would like to point out that the results presented here may be relevant for Ising systems with long range dipolar interactions and two-component BECs.

D.S. thanks DST, India for financial support under the project SP/S2/M-11/2000. The authors thank J. Vidal for useful correspondence.
1 S. Sachdev, Quantum Phase Transitions (Cambridge University Press, Cambridge, 1999).

2 B. K. Chakrabarti, A. Dutta, and P. Sen, Quantum Ising Phases and Transitions in Transverse Ising Models (Springer, Heidelberg, 1996).

3 S. K. Ma Modern Theory of Critical Phenomena (AddisonWesley, New York, 1996).

${ }^{4}$ For a review see, I. Bloch, Nature Physics 1, 23 (2005).

${ }^{5}$ K. Sengupta, S. Powell, and S. Sachdev, Phys. Rev. A 69, 053616 (2004).

6 R. W. Cherng and L. S. Levitov, Phys. Rev. A 73, 043614 (2006).

7 P. Calabrese and J. Cardy, Phys. Rev. Lett. 96, 136801 (2006).

8 D. Gordon and C. M. Savage, Phys. Rev. A 59, 4623 (1999).

9 T. Tomé and M. J. de Oliveira, Phys. Rev A 41, 4251 (1990); A. Krawiecki, Int. J. Mod. Phys. B 19, 4769 (2005); G. M. Buendía and E. Machado, Phys. Rev. E 58, 1260 (1988); M. Keskin, O. Canko, and U. Temizer, Phys. Rev. E 72, 036125 (2005).

10 M. Acharyya, B. K. Chakrabarti, and R. B. Stinchcombe, J. Phys. A 271533 (1994); V. Banerjee, S. Dattagupta, and P. Sen, Phys. Rev. E 521436 (1995).
11 M. Santos and M. J. de Oliveira, Int. J. Mod. Phys. B 13, 2071999.

12 B. K. Chakrabarti and J.-I. Inoue, Ind. J. Phys. 80, 609 (2006); B. K. Chakrabarti, A. Das, and J.-I. Inoue, Euro. Phys. J. B 51, 321 (2006).

13 E. Fradkin, Field Theories of Condensed Matter Systems, Addison-Wesley, Reading, (1991).

14 W. H. Press, S. A. Teukolsky, W. T. Vetterling, and B. P. Flannery, Numerical Recipes in Fortran 7\%: The Art of Scientific Computing (2nd Ed.) (Cambridge University Press, Cambridge, 2001).

15 S. Gasiorowicz, Quantum Physics (John Wiley \& Sons, Singapore, 2000).

16 A. Micheli, D. Jaksch, J.I. Cirac, and P. Zoller, Phys. Rev A 67, 013607 (2003); A. P. Hines, R. H. McKenzie, and G. J. Milburn, Phys. Rev A 67, 013609 (2004).

17 J. Cirac, M. Lewenstein, K. Molmer and P. Zoller, Phys. Rev A 57, 1208 (1998).

18 A. Simoni, F. Ferlaino, G. Roati, G. Modugno, and M. Inguscio, Phys. Rev. Lett 90, 163202 (2003).

19 S. Dusuel and J. Vidal, Phys. Rev. Lett. 93, 237204 (2004); ibid, Phys. Rev. B 71, 224420 (2005); J. Vidal, G. Palacios, and J. Aslangul, Phys. Rev. A 70, 062304 (2004). 